\title{
International Portfolio Optimization with Higher Moments
}

\author{
Maroua MHIRI \\ University of Cergy-Pontoise, THEMA \\ 33, Boulevard du Port, 95011, CERGY-PONTOISE, FRANCE \\ Tel: 33-1-3425-6063 E-mail: Maroua.Mhiri@u-cergy.fr \\ Jean-Luc PRIGENT \\ University of Cergy-Pontoise, THEMA \\ 33, Boulevard du Port, 95011, CERGY-PONTOISE, FRANCE \\ Tel: 33-1-3425-6172Ｅ-mail: jean-luc.prigent@u-cergy.fr
}

\begin{abstract}
We analyze the international portfolio optimization problem by introducing the higher moments of the main financial index returns. We take especially account of their skewness and kurtosis. We introduce various decision criteria, based on these moments. In this framework, we solve different optimization problems: skewness maximization, kurtosis minimization, Polynomial Goal Programming (PGP), and finally, truncated utility maximization. For all of these objective functions, we determine, analyze and compare the optimal solutions, especially their degree of diversification.
\end{abstract}

We illustrate our results on monthly returns of eighteen major international stock market indexes, for the period January 1988 through December 2007.

Keywords: International portfolio diversification, Skewness, Kurtosis, Higher moments, PGP method

JEL classification: C61, G11, G15.

\section{Introduction}

For many decades the prevailing paradigm for asset selection in terms of portfolio strategies has been the seminal Mean-Variance approach developed by Markowitz (1952). However, there is controversy over the issue of whether higher moments should be considered in portfolio selection. (See Samuelson, 1970; Arditti and Levy 1975; Kraus and Litzenberger, 1976; Singleton and Wingender,1986; Prakash et al., 2003, and Sun and Yan 2003). In this paper, we base our portfolio analysis upon the earlier argument that the higher moments of return distributions are relevant to the investor's decision and cannot be neglected, as mentioned in Machina and Müller (1987) and in Jurczenko and Maillet (2006). The objective of this study is threefold. First, the return distributions of 18 international stock markets are tested in context of mean-variance analysis. Second, the skewness and kurtosis are respectively, maximized and minimized under some usual constraints. Finally, portfolio selection with skewness and kurtosis is empirically applied to the sample of international stock markets. Portfolio optimization using higher moments is a more involved problem than standard optimization, such as the mean-variance approach. Indeed, there exists a trade-off between competing and conflicting objectives, i.e., the investor tries to maximize expected return and skewness, while simultaneously minimizing variance and kurtosis. To solve this multi-objective portfolio problem, we have to use specific numerical techniques, since usually no explicit solution can be provided. This study extends the work of Lai et al. (2006) by utilizing Polynomial Goal Programming (PGP), which incorporates investors preferences for higher moments.

This paper is organized as follows. Section 2 summarizes the theory of portfolio selection for various decision rules. In particular, we introduce a criterion based on multiple objectives. Section 3 provides numerical results for various portfolio optimization problems. It discusses the empirical applications of polynomial goal programming and describes the methodology for multi-objective portfolio selection with higher moments. The last section provides the main conclusions. Tables and figures are gathered in Appendix.

\section{Portfolio selection for multiple objective criterion}

In what follows, we search to maximize (resp. minimize) a $p$ th-moment where $p$ is even (resp. odd). It is assumed that the other moments are fixed. We first recall standard results about mean-variance results (see Markowitz, 1959). Then, we detail the skewness maximization and the kurtosis minimization. 


\subsection{Optimization of p-moments}

\subsubsection{Mean-Variance analysis}

Within the mean variance approach of Markowitz (1952), the basic assumption is that risk is measured by variance, and that decision criterion should be to minimize variance given expected return, or to maximize expected return for a given variance. In other words, in this framework, the investment decision is based on the trade-off between higher mean and lower variance of the returns. The locus of optimal mean-variance combinations is called the efficient frontier, on which all rational investors would desire to be positioned.

Introduce some standard notations. In what follows, we denote by $U^{T}$ the transpose of any vector $U$.

Let $R$ be the expected return of the assets:

$$
R=\left(R_{1}, R_{2}, \ldots, R_{n}\right)^{T}
$$

Let $V$ be the variance/covariance matrix:

$$
V=\left[\begin{array}{cccc}
\sigma_{11} & . . & . . & \sigma_{1 n} \\
. . & . . & . . & . . \\
. . & . . & . . & . . \\
\sigma_{n 1} & . . & . . & \sigma_{n n}
\end{array}\right] \text {, with } \sigma_{i j}=\operatorname{cov}\left(R_{i}, R_{j}\right)
$$

The matrix $V$ is assumed to be positive definite.

A portfolio $X$ of asset weights is expressed as:

$$
X=\left(x_{1}, x_{2}, \ldots, x_{n}\right)^{T},
$$

where $x_{i}$ denotes the weight on asset $i$. Note that we have:

$$
X^{T} I=\sum_{i=1}^{n} x_{i}=1
$$

The expected return of a portfolio is given by $E\left[R_{X}\right]=X^{T} E[R]$, and the variance of a portfolio by $\sigma_{X}=X^{T} V X$. Thus, the mean-return of the portfolio $X$ satisfies:

$$
\text { Mean }=R_{X}=X^{T} \bar{R}=\sum_{i=1}^{n} x_{i} \bar{R}_{i},
$$

The variance $V_{X}$ of portfolio return is given by:

$$
V_{X}=X^{T} V X=\sum_{i=1}^{n} x_{i}{ }^{2} \sigma_{i}{ }^{2}+2 \sum_{i=1}^{n} \sum_{j=1, j<i}^{n} x_{i} x_{j} \sigma_{i j},
$$

where $\sigma_{i}^{2}$ is the variance of return $i$, and $\sigma_{i j}$ measures the covariance between returns $i$ and $j$. We denote by

$\sigma_{X}$ the standard deviation of $X$. Often, it is not possible to be short on assets. In that case, we need to add a constraint that all portfolio weights shall be zero or above: $\forall i, x_{i} \geq 0$.

If we search for maximizing the expected return for a given variance, we have to solve the following optimization problem: 


$\begin{cases}\text { Maximize } & X^{T} \bar{R} \\ \text { Subject to } & X^{T} V X=\sigma^{2} \\ & X^{T} I=1 \\ & X \geq 0\end{cases}$

As explained in Markowitz (1952), the portfolio selection problem can be formulated as a quadratic program. We can also search for minimizing the variance. For a portfolio containing $\mathrm{n}$ assets, the minimum variance portfolio is solution of:

$$
\text { (A1) }\left\{\begin{array}{rr}
\text { Minimize } & \sigma_{X}^{2}=\sum_{i=1}^{n} \sum_{j=1}^{n} x_{i} x_{j} \rho_{i j} \sigma_{i} \sigma_{j} \\
\text { Subject to } & x_{1}+x_{2}+\ldots+x_{n}=1
\end{array}\right.
$$

It can be determined by minimizing the following Lagrange function $L$ :

$$
L=\sum_{i=1}^{n} \sum_{j=1}^{n} x_{i} x_{j} \rho_{i j} \sigma_{i} \sigma_{j}+\lambda_{1}\left(1-\sum_{i=1}^{n} x_{i}\right)
$$

in which $\lambda_{1}$ is the Lagrange multiplier, and $\rho_{i j}$ is the correlation coefficient between $R_{i}$ and $R_{j}$.

By using this approach, the minimum variance can also be computed for any given level of expected portfolio return. Adding a condition into Equation (A1) linked to some target expected rate of portfolio return, we get the optimal risky portfolio, by solving:

$$
\text { (A2) }\left\{\begin{array}{lr}
\text { Minimize } & \sigma_{X}^{2}=\sum_{i=1}^{n} \sum_{j=1}^{n} x_{i} x_{j} \rho_{i j} \sigma_{i} \sigma_{j} \\
\text { Subject to } & \sum_{i=1}^{n} x_{i} E\left(R_{i}\right)=E^{*} \\
& \sum_{\mathrm{i}=1}^{\mathrm{n}} \mathrm{x}_{\mathrm{i}}=1
\end{array}\right.
$$

where $E^{*}$ is the target expected return who is determined by the portfolio manager. The Lagrangian objective function can be written as:

$$
L=\sum_{i=1}^{n} \sum_{j=1}^{n} x_{i} x_{j} \rho_{i j} \sigma_{i} \sigma_{j}+\lambda_{1}\left(1-\sum_{i=1}^{n} x_{i}\right)+\lambda_{2}\left(E^{*}-\sum_{i=1}^{n} x_{i} E\left(R_{i}\right)\right)
$$

Taking the partial derivatives of this equation with respect to each of the variables $x_{1}, x_{2}, \ldots, x_{N}, \lambda_{1}, \lambda_{2}$ and setting the resulting equations equal to zero yields the minimization of risk subject to the Lagrangian constraints. Then, we can determine the weights. If there exists a no short selling constraint on the portfolio, we have to add constraints: $x_{i} \geq 0, i=1,2, \ldots, n$.

2.1.2. Skewness maximization under mean and variance constraints

The absolute skewness of the rate of returns $R_{i}$ on assets $i$ are assumed to exist for all risky assets $i$, $i=(1,2, \ldots, n)$. The absolute skewness $S_{a}(x)$ of the portfolio return is defined by: 


$$
S_{a}(x)=E\left[\left(x^{T}(R-\bar{R})\right)^{3}\right]=\sum_{i=1}^{n} x_{i}^{3} s_{i}^{3}+3 \sum_{i=1}^{n}\left(\sum_{j=1, i \neq j}^{n} x_{i}^{2} x_{j} s_{i i j}+\sum_{j=1, i \neq j}^{n} x_{i} x_{j}^{2} s_{i j j}\right)
$$

where $s_{i}^{3}$ is the skewness of asset $i, s_{i i j}$ and $s_{i j j}$ measures the co-skewness (curvilinear interactions), which occur in the joint distribution of $R_{i}$ and $R_{j}$. The relative skewness $S(X)$ is equal to: $S(X)=S_{a}(X) / \sigma_{X}^{3}$.

The second step consists in writing the maximization program under some constraints. We assume that there is no short sale. We fix the levels of mean and variance.

$$
\text { (P2) } \begin{cases}\text { Maximize } & E\left[\left(X^{T}(R-\bar{R})\right)^{3}\right] / \sigma_{X}^{3} \\ \text { Subject to } & X^{T} V X=\sigma^{2} \quad \text { fixed } \\ & X^{T} \bar{R}=R \quad \text { fixed } \\ & X^{T} I=1\end{cases}
$$

2.1.3 Kurtosis minimization under mean and variance constraints

Given the mean and the variance, the absolute kurtosis $K$ is equal to:

$$
\begin{aligned}
\text { Kurtosis } K_{a}(X) & =E\left[\left(X^{T}(R-\bar{R})\right)^{4}\right]=\sum_{i=1}^{n} x_{i}^{4} k_{i}^{4} \\
& +4 \sum_{i=1}^{n}\left(\sum_{j=1}^{n} x_{i}^{3} x_{j} k_{i i i j}+\sum_{j=1}^{n} x_{i} x_{j}{ }^{3} k_{i j j j}\right) \\
& +6 \sum_{i=1}^{n} \sum_{j=1, j \neq i}^{n} x_{i}{ }^{2} x_{j}{ }^{2} k_{i i j j}
\end{aligned}
$$

where $k_{i}^{4}$ is the kurtosis of asset $i, k_{i i i j}, k_{i j j j}$ and $k_{i i j j}$ measure the co-kurtosis, in the joint distribution of $R_{i}$ and $R_{j}$. The relative kurtosis $K$ is given by $K(X)=K_{a}(X) / \sigma_{X}^{4}$.

The second step consists in writing the program under some constraints. We fix the levels of mean and variance and suppose that there is no short sale.

(P3) $\begin{cases}\text { Minimize } & E\left[\left(X^{T}(R-\bar{R})\right)^{4}\right] / \sigma_{X}^{4} \\ \text { Subject to } & X^{T} V X=\sigma^{2} \quad \text { fixed } \\ & X^{T} \bar{R}=R \quad \text { fixed } \\ & X^{T} I=1 \\ & X \geq 0\end{cases}$

\subsection{Portfolio optimization based on Polynomial Goal Programming}

This section extends previous results of Lai et al. (2006) by including and updating more international capital markets. The multi-objective portfolio selection model consists in incorporating the skewness and kurtosis of the return distributions. The mean, variance, and higher moments of the rate of return $R_{i}$ on asset $i$ are assumed to exist for all risky assets $i(i=1,2, \ldots, n)$. The $n \times n$ variance-covariance matrix $V$ of asset rates of return is positive 
definite. The optimal solution consists in selecting the best portfolio weight $X$ such that its multiple objectives are optimized: for example, maximize the expected rate of return, minimize the variance, maximize the skewness and minimize the kurtosis. The best method to find the optimal solution is to aggregate the various objectives into a single objective function. Hence, we use a polynomial goal programming approach to combine these objectives.

Let $d_{1}, d_{2}, d_{3}$ and $d_{4}$ be the goal variables which account for the deviations of expected return, variance, skewness and kurtosis from the aspired levels, $R^{*}, V^{*}, S^{*}$, and $K^{*}$, respectively.

The aspired level indicates the best case scenario for a particular objective without considering other objectives. Hence, the aspired levels, $R^{*}, V^{*}, S^{*}$, and $K^{*}$ can be determined by solving four (independent) sub problems (Q1, Q2, Q3 and Q4).

(Q1) $\begin{cases}\text { Maximize } & R^{*}(x)=X^{T} \bar{R} \\ \text { Subject to } & X^{T} I=1 \\ & X \geq 0\end{cases}$

(Q2) $\begin{cases}\text { Minimize } & V^{*}(x)=X^{T} V X \\ \text { Subject to } & X^{T} I=1 \\ & X \geq 0\end{cases}$

(Q3) $\left\{\begin{array}{ll}\text { Maximize } & S^{*}(x)=E\left[\left(X^{T}(R-\bar{R})\right)^{3}\right] / \sigma_{X}^{3} \\ \text { Subject to } & X^{T} I=1 \\ & X \geq 0\end{array} \quad\right.$ (Q4) $\begin{cases}\text { Minimize } & k^{*}(X)=E\left[\left(X^{T}(R-\bar{R})\right)^{4}\right] / \sigma_{X}^{4} \\ & X \geq 0\end{cases}$

The next step can be accomplished by incorporating investor's preferences for objectives into the construction of a polynomial goal programming problem. Consequently, portfolio selection with higher moments is a solution of PGP, and multiple objectives can be achieved. The PGP method was first introduced by Tayi and Leonard (1988) in order to facilitate bank balance sheet management. It has subsequently been used by Lai (1991), Chunhachinda et al. (1997), Sun and Yan (2003), and Prakash et al. (2003) to solve portfolio selection problems involving a significant degree of skewness. We increase the dimensionality of the PGP portfolio selection problem from mean-variance-skewness to mean-variance-skewness-kurtosis to can take account of the non-normality of returns. The PGP model allows the determination of asset allocation, by solving the following problems:

(i) Which assets should be included?

(ii) How much capital should be allocated in each asset?

(P4) $\begin{cases}\text { Minimize } Z=\left|\frac{d_{1}}{R^{*}}\right|^{\lambda_{1}}+\left|\frac{d_{2}}{V^{*}}\right|^{\lambda_{2}}+\left|\frac{d_{3}}{S^{*}}\right|^{\lambda_{3}}+\left|\frac{d_{4}}{K^{*}}\right|^{\lambda_{4} 4} \\ \text { subject to } \quad X^{T} \bar{R}+d_{1}=R^{*} \\ & X^{T} V X-d_{2}=V^{*} \\ & E\left(X^{T}(\tilde{R}-\bar{R})\right)^{3} / \sigma^{3}+d_{3}=S^{*} \\ & E\left(X^{T}(\tilde{R}-\bar{R})\right)^{4} / \sigma^{4}-d_{4}=K^{*} \\ & X^{T} I=1 \\ & X \geq 0 \\ & d_{i} \geq 0, i=1, \ldots, 4\end{cases}$


As explained in Lai (1991), solving the (PGP) problem is based on a two-step procedure. First, the aspired levels of $R^{*}, V^{*}, S^{*}$ and $K^{*}$ for the expected return, variance, skewness and kurtosis, respectively, are deduced from $(Q 1-Q 4)$. Then, these aspired values are substituted into (P4). The minimum value of $Z$ can be found for a given set of investor preferences $\left\{\lambda_{i}\right\}(i=1,2,3,4)$, since the solutions of the sub problems $(Q 1-Q 4)$ are at least as good as the solution linked to (P4), where all objectives $R, V, S$ and $K$ are considered simultaneously. The values of the goal variables $d_{1}, d_{2}, d_{3}$ and $d_{4}$ are always non-negative. They represent the amount of underachievement with respect to the best scenario.

\subsection{Maximization of a polynomial utility}

We suppose now that the skewness and the kurtosis are absolute. We consider an investor who allocates her portfolio to maximize the expected utility $U(W)$. To assess the importance of higher moments on the asset allocation, we approximate the expected utility by a Taylor series expansion truncated at order 4 (see Machina and Müller, 1987).

We get:

$U(W) \approx U(\bar{W})+U^{\prime}(\bar{W})(W-\bar{W})+\frac{U^{\prime \prime}(\bar{W})}{2 !}(W-\bar{W})^{2}+\frac{U^{\prime \prime \prime}(\bar{W})}{3 !}(W-\bar{W})^{3}+\frac{U^{\prime \prime \prime \prime}(\bar{W})}{4 !}(W-\bar{W})^{4}$

Then, taking expectation of both terms, we deduce:

$$
E(U(W)) \approx U(\bar{W})+\frac{U^{\prime \prime}(\bar{W})}{2 !} \operatorname{Var}(W)+\frac{U^{\prime \prime \prime}(\bar{W})}{3 !} \text { Skew }_{a b s}(W)+\frac{U^{\prime \prime \prime \prime}(\bar{W})}{4 !} \operatorname{Kurto}_{a b s}(W)
$$

Hence, we also include a simpler method of multi-moment optimization as a comparison. This approach takes the form:

$$
\begin{aligned}
& \text { (P5) } \begin{cases}\text { Maximize } & \bar{R}-a \text { Var }+ \text { bSkew }_{\text {absolute }}-\text { cKurto }_{\text {absolute }} \\
\text { subject to } & X^{T} I=1 \\
& X \geq 0 \\
& a, b, c>0\end{cases} \\
& \text { with } \quad a=-\frac{U^{\prime \prime}(\bar{W})}{2 !} ; \quad b=\frac{U^{\prime \prime \prime}(\bar{W})}{3 !} ; \quad \text { and } \quad c=-\frac{U^{\prime \prime \prime}(\bar{W})}{4 !}
\end{aligned}
$$

For example, consider a CRRA utility function given by:

$$
U(x)=\frac{x^{1-\gamma}}{1-\gamma}, \text { with } \gamma>1
$$

Here $\gamma$ denotes the relative risk aversion. We get:

$$
a=\frac{\gamma(\bar{X})^{-(\gamma+1)}}{2 !}, b=\frac{\gamma(\gamma+1)(\bar{X})^{-(\gamma+2)}}{3 !}, \text { and } \mathrm{c}=\frac{\gamma(\gamma+1)(\gamma+2)(\bar{X})^{-(\gamma+3)}}{4 !}
$$

\section{Empirical analysis}

\subsection{Statistical data analysis}

The sample data (Note 1) consists in monthly rates of return of 18 international stock market indexes for various developed markets in North America, Asia, Australia and Europe and emerging markets in Latin America, Asia and Africa for the period January 1988 through December 2007. The five emerging markets indices included in this study are those of Mexico, Hong Kong, Malaysia, Singapore, and South Africa. The nine European indices include those of Belgium, Denmark, France, Germany, Italy, Spain, Sweden, Switzerland and the UK. From Asia, we include the Japan as the only developed market. The two North American markets are Canada and US. The inclusion of the index of the Australia adds up to a total of eighteen countries in our sample. The data reports the international price indices as covered into US dollars at the appropriate period's spot foreign exchange rate. It 
means that we examine mainly the US investor's portfolio. Holding period returns for each country index $(j)$ are calculated as follows:

$$
R_{j, t}=\frac{P_{j, t} X_{j, t}-X_{j, t-1} P_{j, t-1}}{X_{j, t-1} P_{j, t-1}},
$$

where:

$P_{j, t}$ is the price of the stock index in country $j$ traded at time $t$.

$X_{j, t}$ is the country $\mathrm{j}$ exchange rate at time $\mathrm{t}$, expressed in US dollars.

$R_{j, t}$ is the exchange-rate-adjusted rate of return from investment in country $j$ 's stock at time $t$.

The first four moments of all indices are provided in Table (1) (see Appendix B). For preliminary analysis, Table (1) first lists the means and the variances of the rates of returns of the 18 international stock markets. Looking at the first column reveals that Mexico has the highest mean rate of return (1.95\%), followed by Malaysia (1.24\%) and Hong Kong (1.22\%). Japan, Italy and UK provide the lowest means for monthly investment horizon. We note that none market has a negative mean return. In column 3, the evidence indicates that the market with highest return but with highest risk is Mexico: indeed, it has the highest variance of returns $(0.87 \%)$, while the US has the lowest $(0.14 \%)$, followed by the UK $(0.19 \%)$.

Table (1) also provides the values of skewness and kurtosis for each of the indices returns. International markets exhibits a negative skewness except for Australia, Belgium, Hong Kong, Italy, Japan, Malaysia and Singapore. We note also that Singapore has the highest skewness (0.757) while Canada has the lowest $(-0.53)$. Singapore has the highest Kurtosis (9.90) and Denmark has the lowest (3.123).

\subsection{Numerical illustrations for each criterion}

In what follows, we give the numerical solutions of each of optimization problems detailed in Section 2.

\subsubsection{Mean-variance criterion}

\section{[Insert Figure 1]}

This frontier shows as usual that the standard deviation risk increases when mean return increases. The minimum risk is $3.35 \%$. The expected return is varying between $0.83 \%$ and $1.95 \%$. The Mean-Variance Portfolio (MVP) is defined as the portfolio having the minimum standard deviation. The MVP is the portfolio chosen by the most risk averse investor (infinite aversion to variance). The MVP results from investing approximately $46 \%$ in US asset, 23\% in Australian asset, and 12\% in UK asset. The MVP has the following characteristics (monthly returns):

$$
\bar{R}=0.008314 ; \sigma=0.033547
$$

However, various constraints may preclude a particular investor from choosing portfolios on the efficient frontier. Short sale restrictions are possible constraints. Short sale is a usual condition on market transactions.

Suppose that we have two assets: asset $A$ and asset $B$ with $E\left(R_{A}\right) \geq E\left(R_{B}\right)$ and $\sigma_{A} \geq \sigma_{B}$.

Now, relaxing the assumption of no short selling, investors could sell the lowest-return asset $B$. If the number of short sales is unrestricted, then by a continuous short selling of $B$ and reinvesting in $A$ the investor could generate an infinite expected return. The upper bound of the highest-return portfolio would no longer be A but infinity. Likewise the investor could short sell the highest-return security $A$ and reinvest the proceeds into the lowest-yield security $B$, thereby generating a return less than the return on the lowest-return assets. Given no restriction on the amount of short selling, an infinitely negative return can be achieved, thereby removing the lower bound of $B$ on the efficient frontier. Hence, short selling generally will increase the range of investment strategies from the minimum-variance portfolio to plus or minus infinity.

If we take again our sample of 18 index prices, we suppose that we are short on US asset and long on the Mexican asset. The choice of US index and Mexico index is not arbitrary, but is a rational choice because the US index is the lowest risky asset (whose variance is equal at 1.459) and the Mexico index has the highest risk $\left(\sigma^{2}=8.69\right)$ but also the highest return (1.95\%). By using this strategy, the investor could sell the lowest risk and reinvesting in Mexico index and generate an infinite expected return. In our case, we choose that the minimum asset weight $=-100 \%$ and maximum asset weight $=100 \%$.

The efficient frontier of unconstrained portfolio is displayed in Figure 2. 


\section{[Insert Figure 2]}

Figure 2 shows that the Mean-Variance efficient frontier of unrestricted portfolios dominates the Mean-Variance efficient portfolio of restricted portfolios: the highest return of dominant curve is equal at 0.023226 and has a risk equal to 0.11419 . Figure 3 illustrates the efficient frontiers when a riskless asset $(0.8 \%)$ is available on the market.

[Insert Figure 3]

\subsubsection{Skewness maximization}

First, we examine skewness values for all efficient portfolios. As shown in Figure 4, all these skewness are negative. [Insert Figure 4]

The maximum skewness value for mean-variance efficient portfolios is the MVP portfolio itself $\left(S k_{M V P}=-0.0672917\right)$.

Now, we examine relations between mean, variance and skewness. For this purpose, we provide the shape of the skewness for all portfolios in Figure 6. We indicate simultaneously its projection on the standard deviation/mean return plane, which corresponds to the set of all portfolios delimited by the Mean-Variance efficient frontier.

\section{[Insert Figure 6]}

The maximum skewness of all portfolios is reached for $\bar{R}=0.00971 ; \sigma=0.093233 ; S k=0.87409$.

The maximum skewness is achieved by a combination of about $70 \%$ on Singapore index, which has the highest skewness $(0.757)$ and $30 \%$ on Hong-Kong index, which has also a positive skewness (0.313).This is due to positive coskewness.

\subsubsection{Kurtosis minimization}

We begin by looking at kurtosis values for all efficient portfolios.

[Insert Figure 5]

The minimum kurtosis value for mean-variance efficient portfolios is also the MVP portfolio kurtosis $\bar{R}=0.0083184 ; \sigma=0.033547 ;$ Kurtosis $=3.892$.

Now, we examine relations between mean, variance and kurtosis. We indicate the shape of the kurtosis for all portfolios in Figure 7, together with its projection on the standard deviation/mean return plane.

[Insert Figure 7]

The minimum kurtosis value for all portfolios is given by $\bar{R}=0.0083184 ; \sigma=0.06973 ; K=0.069076$.

This result shows that diversification allows to minimize kurtosis, due to negative cokurtosis.

\subsubsection{The four moments control}

In this section, our main objective is to determine the investor's portfolio allocation within the four-moment framework. This can be done by incorporating investor's preferences. In what follows, we detail the methodology corresponding to Problem (P4), based on the Polynomial Goal Programming.

First of all, we use:

1) The variances and the covariances for the monthly rates of return.

2) The skewness and co-skewness for monthly rates of returns.The sizes and signs of the coskewness will vary depending upon the degree of curvilinear relationship between the two markets.

3) The values of kurtosis and co-kurtosis for monthly rates of returns.

Second, by solving the four sub problems Q1, Q2, Q3 and Q4, we can obtain the aspired levels of the first four moments as shown in Table (2) (see Appendix B). In order to verify the effect of investors preferences on portfolio selection, different levels of preferences are investigated. Specifically, investors' preferences of $(3,1,1,0)$, $(3,1,2,1),(3,1,3,1),(1,3,1,1),(1,1,1,3),(1,3,1,3),(1,2,3,2),(3,1,2,3)(2,3,3,1)$ and $(1,1,0,0)$ are included in our experiment. The results based on the preference structure of $(3,1,1,0),(3,1,2,1)$ and $(3,1,3,1)$ imply that the investors are willing to pursue more excess returns regardless of risk level while those of $(1,3,1,1)$, $(1,1,1,3)$ and $(1,3,1,3)$ give more emphasis on risk control. However, the investors based on the preference of $(1$, $2,3,2),(3,1,2,3)$ and $(2,3,3,1)$ consider several objectives simultaneously.(1, 1, 0, 0) is a benchmark case, representing the Markowitz mean-variance portfolio. Detailed results are presented in middle part of Table (3) (see Appendix B). 
Similarly, investor's preferences also affect the four moments related to portfolios. The last part of Table (3) clearly shows that different combinations of investors' preferences on expected return $\left(\lambda_{1}\right)$, variance $\left(\lambda_{2}\right)$, skewness $\left(\lambda_{3}\right)$ and kurtosis $\left(\lambda_{4}\right)$ lead to optimal portfolios with substantially different moment characteristics.

The more importance investors' preferences attach to a certain moment, i.e., the greater the preference parameter for this moment, the more favourable value of this moment statistic would be in the optimal portfolio. That is, as a result of the trade-off between the four moments, at least one of the other three moment statistics deteriorates.

For instance, the highest skewness is achieved in Portfolio A. It has not only higher preference parameter over skewness $\left(\lambda_{3}=3\right)$, but also does not incorporate preference for kurtosis $\left(\lambda_{4}=0\right)$. Consequently, the kurtosis is very important. The same pattern is observed for Portfolio B. In portfolio $\mathrm{C}$ and $\mathrm{D}$, we inverse the scenario: the investor has a preference for the kurtosis and neglect the skewness. The other cases are a trade-off between mean, variance, skewness and kurtosis. The last case in a mean-variance efficient portfolio. The empirical findings suggest that the incorporation of higher moment into an investor's portfolio decision causes a major change in the construction of the optimal portfolio.

In portfolio A1, investor's higher preference for expected return leads to higher expected return than that in portfolio A, resulting in a lower skewness than portfolio A. Thus, as the investor preference for expected returns increases, she must settle for lower skewness. Compared to Portfolio B1, a higher preference for skewness leads to a higher portfolio skewness but also a higher portfolio kurtosis than Portfolio B. Similarly, we also consider changing the preference parameters of Portfolio $\mathrm{C}$ from $(1,1,0,3)$ to $(2,1,0,3)$ while holding the values of variance and skewness constant. This result in the mean rose from 9.769 to 9.778 , and the kurtosis rose from 0.88 to 0.89 . Thus, as preference for expected returns increases, the investors must settle for higher kurtosis, holding skewness and variance are held constant. That is, higher preference for skewness leads to higher skewness, but also higher kurtosis. Likewise, when skewness and kurtosis are held constant, as shown in Portfolio D and D1, higher preference for expected return leads to higher returns, but also higher variance, which is the same to traditional Markowitz's mean variance model. Therefore, expected return, variance, skewness, and kurtosis are conflicting objective in portfolio diversification and risk averse investors prefer portfolios with high skewness and low kurtosis, thus requiring a lower required rate of return.

3.2.5 The polynomial utility

We examine the numerical solution of Program (P5). We consider three basic values for the relative risk aversion $\gamma:$

$-\gamma=2$ : the investor is rather aggressive;

$-\gamma=5$ : the investor is moderate;

$-\gamma=10$ : the investor is rather conservative.

In the case of $\gamma=2$ (P5) can be write as:

$\bar{R}-0.963 \mathrm{Var}+0.95$ Skew $_{\text {absolute }}-0.938$ Kurto absolute .

For $\gamma=5$, the objective function is equal to:

$\bar{R}-2.282$ Var +4.496 Skew $_{\text {absolute }}-7.749$ Kurto $_{\text {absolute }}$.

In the last case $\gamma=10$, the objective function is given by:

$\bar{R}-4.565$ Var +16.603 Skew $_{\text {absolute }}-49.806$ Kurto $_{\text {absolute }}$.

The virtue of Equation (P5) is that it incorporates the same multi-moment analysis but in a much simpler approach. Program (P5) states that the goal is to maximize the expected return of the portfolio while trying to minimize the impacts of volatility and kurtosis and maximize the positive benefits of skewness. Essentially, Program (P5) is an expanded version of a mean-variance utility function. The weights $a, b$, and $c$ can be set to pre-determined levels depending upon an investor's tolerance for greater volatility, skewness and kurtosis.

This case is the same in PGP when the investors' preference of are equal, i.e. $\lambda_{1}=\lambda_{2}=\lambda_{3}=\lambda_{4}=1$. It is worth noting that preference set $(1,1,1,1)$ was a compromise case where the weights for mean, variance, skewness, and kurtosis are equivalent. Note that US weights $\left(w_{18}\right)$ are quite different according to decision criterion: for example, for the mean-variance analysis, it is about $46 \%$, for the skewness maximization, it is equal to $0 \%$ and for the utility criterion, it is about $13 \%$ (see also Table 3 ). 


\section{Conclusion}

This study shows the importance of choice criterion when dealing with international portfolio diversification. We begin by examining mean-variance analysis in case of restricted and unrestricted portfolio. In second step, the investor preferences are incorporated in maximization of the skewness and a minimization of the kurtosis. Then we introduce PGP optimization function. This approach allows us to solve for multiple competing and conflicting portfolio objectives based on mean-variance-skewness-kurtosis framework. Finally, we consider utility maximization. Our empirical analysis shows the PGP approach can efficiently solve portfolio problem with multiple conflicting objectives and can find optimal portfolio and make the corresponding investment decisions. The empirical findings suggest that the incorporation of skewness into an investor's portfolio decision causes a major change in the resultant optimal portfolio, as shown by the various US weights depending on decision criterion.

\section{References}

Arditti F. D. and Levy H. (1975). Portfolio efficiency analysis in three moments: The multiperiod case. Journal of Finance, 30, 797-809.

Chunhachinda P., Dandapani K., Hamid S. and Prakash A.J. (1997). Portfolio selection and skewness: Evidence from international stock markets. Journal of Banking \& Finance, 21, 143-167.

Jurczenko E. and Maillet B. (2006). Multi-Moment Asset Allocation and Pricing Models. Wiley.

Kraus A. and Litzenberger R. (1976). Skewness preference and the valuation of risk assets. Journal of Finance, 33, 303-310.

Lai T. (1991). Portfolio selection with skewness. Review of Quantitative Finance and Accounting, 1, 293-305.

Lai K.K, Yu L, and Wang S. (2006). Mean-variance-skewness-kurtosis-based portfolio optimization. Proceedings of the First International Multi-Symposiums on Computer and Computational Sciences - Volume 2 (IMSCCS'06) pp 292-297.

Machina M. and Müller S. M. (1987). Moment preferences and polynomial utility. Economics Letters, 23, 349-353

Markowitz H. (1952). Portfolio selection. Journal of Finance, 7, 77-91.

Markowitz H. (1959). Portfolio Selection: Efficient Diversification of Investments. Wiley: New York.

von Neumann, J. and Morgenstern O. (1944). Theory of Games and Economic Behaviour. Princeton University Press.

Prakash, A.J., Chang C.-H. and Pactwa T.E. (2003). Selecting a portfolio with skewness: Recent evidence from US, European, and Latin American equity markets. Journal of Banking \& Finance, 27, 1375-1390.

Samuelson P. (1970). The fundamental approximation theorem of portfolio analysis in terms of means, variances and higher moments. Review of Economic Studies, 25, 65-86.

Singleton J. and Wingender J. (1986). Skewness persistence in common stock returns. Journal of Financial and Quantitative Analysis, 13, 335-341.

Sun Q. and Yan Y. (2003). Skewness persistence with optimal portfolio selection. Journal of Banking \& Finance, 27, 111-112.

Notes

Note 1. The data source for these indices is Datastream. 
Table 1. Statistical analysis of indices

\begin{tabular}{llllll}
\multicolumn{2}{r}{ Mean $\left(* 10^{-3}\right)$, Variance $\left(* 10^{-3}\right)$, Relative Skewness, Relative Kurtosis } \\
\hline \hline Countries & & $R$ & $\sigma^{2}$ & \multicolumn{1}{c}{ Skew } & Kurto \\
\hline \hline Australia & 1 & 9.806 & 2.864 & 0.063 & 3.941 \\
Belgium & 2 & 7.969 & 3.138 & 0.072 & 3.56 \\
Canada & 3 & 9.686 & 2.218 & -0.53 & 4.63 \\
Denmark & 4 & 11.93 & 3.827 & -0.127 & 3.123 \\
France & 5 & 8.363 & 3.354 & -0.136 & 3.765 \\
Germany & 6 & 8.839 & 2.822 & -0.376 & 4.254 \\
Hong Kong & 7 & 12.24 & 5.834 & 0.313 & 4.897 \\
Italy & 8 & 6.203 & 4.071 & 0.155 & 3.561 \\
Japan & 9 & 1.983 & 4.436 & 0.454 & 4.453 \\
Malaysia & 10 & 12.38 & 6.544 & 0.423 & 8.76 \\
Mexico & 11 & 19.48 & 8.692 & -0.262 & 4.189 \\
Singapore & 12 & 8.626 & 4.595 & 0.757 & 9.906 \\
South Africa & 13 & 10.58 & 5.533 & -0.403 & 5.384 \\
Spain & 14 & 9.419 & 4.377 & -0.122 & 3.945 \\
Sweden & 15 & 11.26 & 4.772 & -0.137 & 3.311 \\
Switzerland & 16 & 10.39 & 3.163 & -0.313 & 3.889 \\
U.K & 17 & 6.948 & 1.916 & -0.023 & 3.317 \\
U.S & 18 & 8.567 & 1.459 & -0.273 & 3.687 \\
\hline \hline
\end{tabular}

Table 2. The aspired levels of four moments

\begin{tabular}{ccccc}
\hline \hline Sub-objectives & $R^{*}(x)$ & $V^{*}(x)$ & $S^{*}(x)$ & $K^{*}(x)$ \\
\hline optimal values & $19.484 * 10^{-3}$ & $1.125 * 10^{-3}$ & 0.874 & 0.069 \\
\hline \hline
\end{tabular}

Table 3. Asset allocation and moment statistics for different preferences

\begin{tabular}{|c|c|c|c|c|c|c|c|c|c|c|}
\hline Portfolio & $\mathrm{A}$ & B & $\mathrm{C}$ & $\mathrm{D}$ & $\bar{E}$ & $\bar{F}$ & $\bar{G}$ & $\mathrm{H}$ & 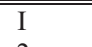 & 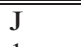 \\
\hline$\lambda_{1}$ & 1 & 3 & 1 & 1 & 3 & 1 & 1 & 3 & 2 & 1 \\
\hline$\lambda_{2}$ & 1 & 1 & 1 & 3 & 1 & 3 & 2 & 1 & 3 & 1 \\
\hline$\lambda_{3}$ & 3 & 1 & 0 & 0 & 3 & 1 & 3 & 2 & 3 & 0 \\
\hline$\lambda_{4}$ & 0 & 0 & 3 & 1 & 1 & 3 & 2 & 3 & 1 & 0 \\
\hline Australia & 0.301 & 0.27 & 0.115 & 0.077 & 0.087 & 0.117 & 0.109 & 0.123 & 0.08 & 0.239 \\
\hline Belgium & 0.024 & 0.076 & 0.059 & 0.053 & 0.055 & 0.054 & 0.057 & 0.061 & 0.054 & 0.021 \\
\hline Canada & 0.012 & 0.045 & 0.058 & 0.046 & 0.045 & 0.049 & 0.044 & 0.051 & 0.044 & 0.038 \\
\hline Denmark & 0 & 0.039 & 0.06 & 0.059 & 0.057 & 0.065 & 0.057 & 0.056 & 0.058 & 0.031 \\
\hline France & 0 & 0 & 0.028 & 0.047 & 0.045 & 0.03 & 0.039 & 0.032 & 0.047 & 0 \\
\hline Germany & 0 & 0.001 & 0.058 & 0.053 & 0.049 & 0.046 & 0.042 & 0.047 & 0.048 & 0 \\
\hline HongKong & 0 & 0 & 0.025 & 0.049 & 0.049 & 0.049 & 0.051 & 0.034 & 0.053 & 0 \\
\hline Italy & 0.047 & 0.062 & 0.069 & 0.066 & 0.071 & 0.067 & 0.076 & 0.078 & 0.069 & 0.033 \\
\hline Japan & 0.080 & 0.054 & 0.07 & 0.069 & 0.079 & 0.057 & 0.087 & 0.088 & 0.076 & 0 \\
\hline Malaysia & 0 & 0.047 & 0.058 & 0.051 & 0.049 & 0.055 & 0.048 & 0.051 & 0.049 & 0.044 \\
\hline Mexico & 0 & 0.032 & 0.092 & 0.102 & 0.091 & 0.11 & 0.087 & 0.081 & 0.096 & 0.03 \\
\hline Singapore & 0.377 & 0 & 0.014 & 0.03 & 0.032 & 0.024 & 0.036 & 0.025 & 0.034 & 0 \\
\hline SouthAfrica & 0 & 0.011 & 0.039 & 0.044 & 0.043 & 0.044 & 0.042 & 0.038 & 0.044 & 0.002 \\
\hline Spain & 0 & 0 & 0.031 & 0.044 & 0.041 & 0.032 & 0.035 & 0.03 & 0.043 & 0 \\
\hline Sweden & 0 & 0 & 0.021 & 0.051 & 0.044 & 0.031 & 0.029 & 0.017 & 0.049 & 0 \\
\hline Switzerland & 0 & 0.021 & 0.049 & 0.047 & 0.045 & 0.046 & 0.041 & 0.043 & 0.045 & 0 \\
\hline UK & 0 & 0.093 & 0.08 & 0.062 & 0.063 & 0.062 & 0.061 & 0.073 & 0.059 & 0.098 \\
\hline US & 0.158 & 0.249 & 0.074 & 0.051 & 0.053 & 0.064 & 0.059 & 0.071 & 0.05 & 0.462 \\
\hline Mean & 8.321 & 8.971 & 9.77 & 1010.02 & 9.783 & 10.19 & 9.645 & 9.481 & 99.9 & 9.263 \\
\hline Variance & 1.768 & 1.873 & 1.44 & 1.589 & 1.556 & 1.52 & 1.517 & 1.442 & 1.589 & 1.157 \\
\hline Skewness & 0.162 & -0.081 & -0.051 & -0.044 & -0.039 & -0.045 & -0.034 & -0.039 & -0.039 & -0.17 \\
\hline Kurtosis & 2.156 & 0.48 & 0.88 & 0.07 & 0.071 & 0.081 & 0.077 & 0.087 & 0.07 & 1.267 \\
\hline
\end{tabular}


Table 4. Moment statistics according to various preferences

\begin{tabular}{ccccccccc}
\hline \hline Portfolio & A & A1 & B & B1 & C & C1 & D & D1 \\
& & & & & & & & \\
$\lambda_{1}$ & 1 & 2 & 3 & 3 & 1 & 2 & 1 & 2 \\
$\lambda_{2}$ & 1 & 1 & 1 & 1 & 1 & 1 & 3 & 3 \\
$\lambda_{3}$ & 3 & 3 & 1 & 2 & 0 & 0 & 0 & 0 \\
$\lambda_{4}$ & 0 & 0 & 0 & 0 & 3 & 3 & 1 & 1 \\
\hline \hline & & & & & & & & \\
Mean & 8.321 & 8.404 & 8.971 & 8.937 & 9.769 & 9.778 & 10.01 & 10.02 \\
Variance & 1.768 & 1.772 & 1.873 & 1.242 & 1.44 & 1.44 & 1.588 & 1.589 \\
Skewness & 0.162 & 0.16 & -0.08 & -0.051 & -0.05 & -0.05 & -0.044 & -0.044 \\
Kurtosis & 2.156 & 2.183 & 0.351 & 0.48 & 0.088 & 0.089 & 0.0695 & 0.0695 \\
\hline \hline
\end{tabular}

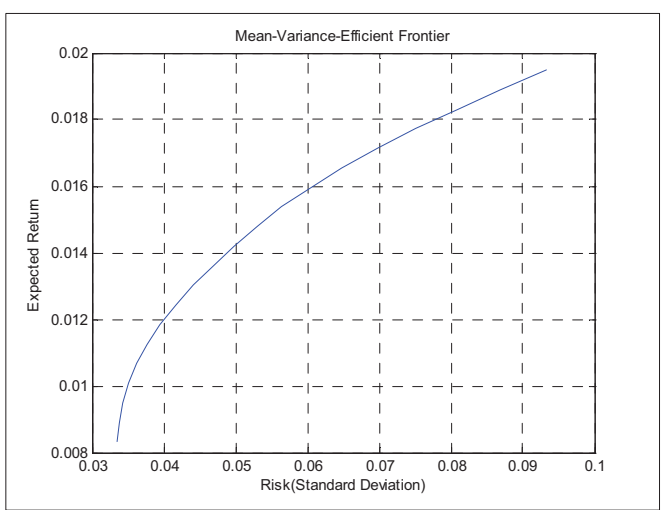

Figure 1. The mean-variance efficient frontier

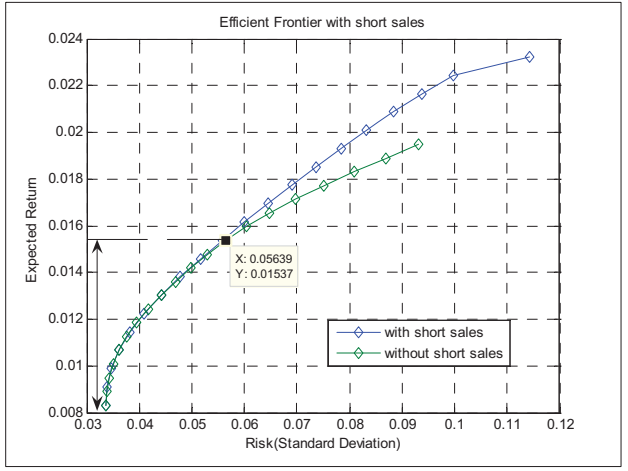

Figure 2. The efficient frontier of unrestricted/restricted portfolios

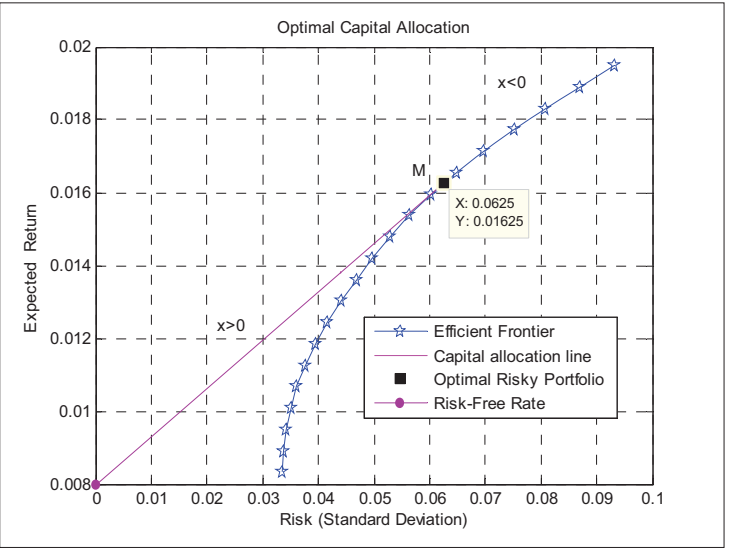

Figure 3. The efficient frontier with a risk-free asset 


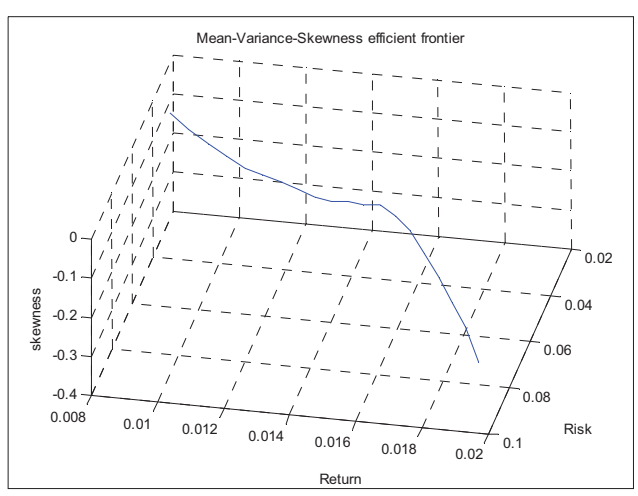

Figure 4. Skewness for Mean-Variance efficient frontier

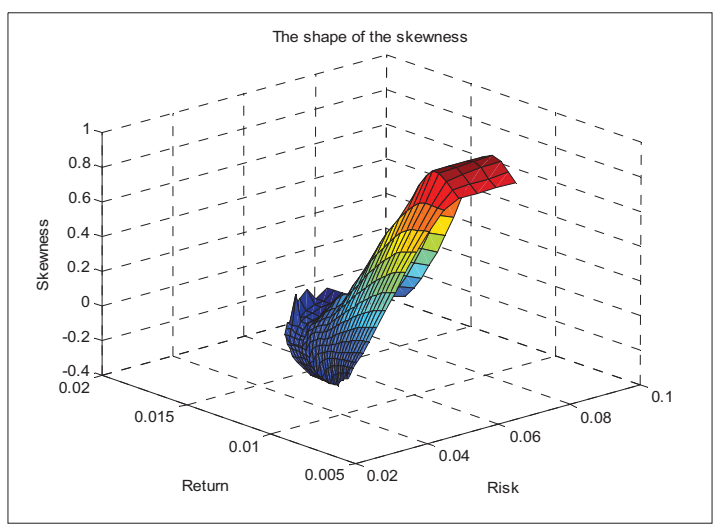

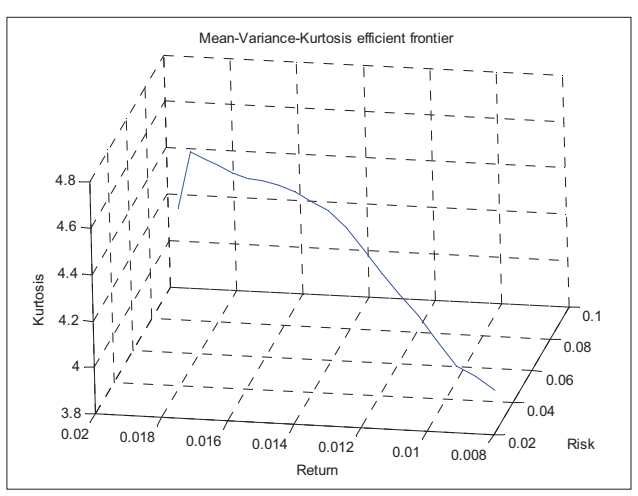

Figure 5. Kurtosis for Mean-Variance efficient frontier

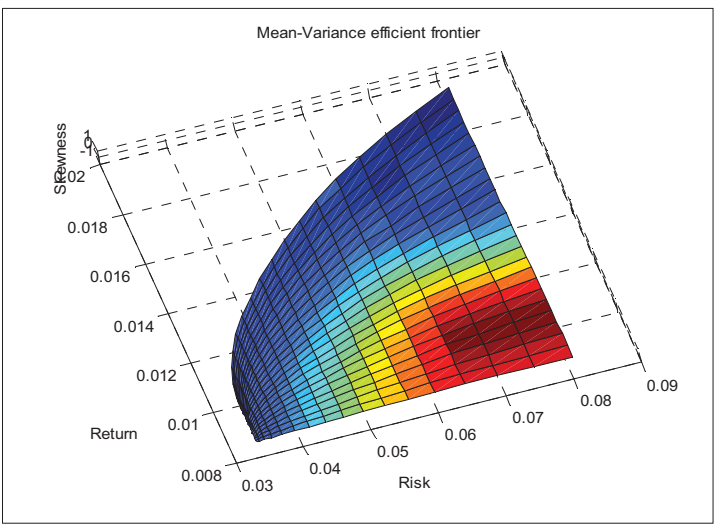

Figure 6. Shape of the Skewness
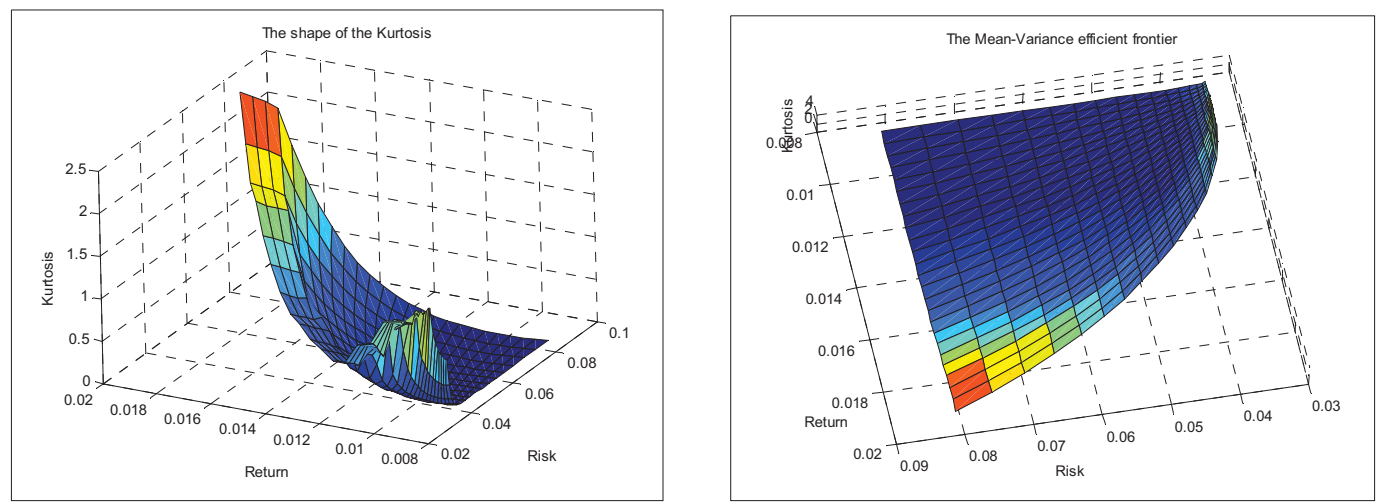

Figure 7. Shape of the Kurtosis 